\title{
Surface formation routes of interstellar molecules: hydrogenation reactions in simple ices
}

\author{
S. Ioppolo • H. M. Cuppen • H. Linnartz
}

Received: 7 March 2011/Accepted: 22 June 2011/Published online: 14 July 2011

(C) The Author(s) 2011. This article is published with open access at Springerlink.com

\begin{abstract}
It has been a long standing problem in astrochemistry to explain how molecules can form in a highly dilute environment such as the interstellar medium. In recent years it has become clear that not only ion/radical-molecule gas-phase reactions, but also solid state reactions on icy dust grains play an important role in the formation of new species. In order to investigate the underlying processes, laboratory based experiments are needed to simulate surface reactions induced by photon (UV) processing or particle (atom, cosmic ray, electron) bombardment of interstellar ice analogs. Here, the latest research performed on SURFace REaction SImulation DEvice (SURFRESIDE), one of the ultra-high vacuum setups in the Sackler Laboratory for Astrophysics in Leiden is reviewed. The focus is on hydrogenation, i.e., H-atom addition reactions in interstellar ice analogs for astronomically relevant temperatures. We discuss how molecules form when $\mathrm{CO}$ and $\mathrm{O}_{2}$ containing ices are exposed to thermal hydrogen atoms under fully controlled experimental conditions. Surface formation schemes for interstellar relevant species, such as solid methanol, water, and carbon dioxide are investigated and chemical links between molecular species in space are discussed.
\end{abstract}

Keywords Astrochemistry · Infrared: ISM - ISM: atoms · ISM: molecules · Methods: laboratory

This paper is one of those collected in this issue and presented on the occasion of the symposium "Astrochemistry: molecules in space and time" (Rome, 4-5 November 2010), sponsored by Fondazione "Guido Donegani" and the Accademia Nazionale dei Lincei. Other 11 related papers and an Introduction to the whole series of papers on this topic were published in the previous issue of Rendiconti Lincei (Vol. 22-2, June 2011).

S. Ioppolo $\cdot$ H. Linnartz $(\bowtie)$

Raymond and Beverly Sackler Laboratory for Astrophysics, Leiden Observatory,

Leiden University, PO Box 9513, 2300 RA Leiden, The Netherlands

e-mail: linnartz@strw.leidenuniv.nl

H. M. Cuppen

Institute for Molecules and Materials (IMM), Radboud University Nijmegen,

PO Box 9010, 6500 GL Nijmegen, The Netherlands 


\section{Introduction}

Our Galaxy is largely empty. By terrestrial standards the space between stars can be considered as a near-perfect vacuum: the average particle density in the solar neighborhood is roughly a factor of $10^{19}$ less than in the terrestrial atmosphere at sea level. Nevertheless, the highly diluted material present between the stars, the interstellar medium (ISM), plays a central role in the chemical evolution of our Galaxy. The ISM is the repository of ashes from previous generations of stars and it is itself the birthplace of new stars and planetary systems.

The interstellar matter consists of about $99 \%$ gas, mainly hydrogen, helium and some heavier elements (e.g., C, O, N, S), and 1\% (sub)micron size silicate and carbonaceous dust grains by mass. The identification of rotational, vibrational, and electronic spectra has established the presence of a large variety of polyatomic molecules, ions and radicals in the ISM, both in the gas phase and in the solid state. In fact, over 150 different molecular species (excluding isotopomers) have been assigned. The spectra are probes of the physical conditions and chemical history of the regions in space where molecular species reside. These species include a variety of inorganic compounds (e.g., $\mathrm{H}_{2} \mathrm{O}, \mathrm{CO}, \mathrm{CO}_{2}, \mathrm{NH}_{3}$ and $\mathrm{SO}_{2}$ ), organics (e.g., $\mathrm{CH}_{4}, \mathrm{H}_{2} \mathrm{CO}, \mathrm{CH}_{3} \mathrm{OH}, \mathrm{HCOOH}$, and $\mathrm{CH}_{3} \mathrm{CH}_{2} \mathrm{OH}$ ), ions (e.g., $\mathrm{HCO}^{+}$ and $\mathrm{C}_{6} \mathrm{H}^{-}$) and species identified only in ice (e.g., $\mathrm{OCN}^{-}$and $\mathrm{NH}_{4}^{+}$), as well as unsaturated hydrocarbon chains (e.g., $\mathrm{HC}_{n} \mathrm{~N}$ with $n$ as large as $n=11$ ) (Tielens 2005). Recently, also the fullerenes $\mathrm{C}_{60}$ and $\mathrm{C}_{70}$ were unambiguously detected (Cami et al. 2010; Sellgren et al. 2010). Aromatic species such as polycyclic aromatic hydrocarbons (PAHs) are likely present in space, but not included in the count, since they have not been uniquely identified yet.

For the low temperatures in space, gas-phase molecules freeze onto the surface of dust grains that act as $\mu \mathrm{m}$ large cryopumps. The presence of ice in the cold and dense interstellar medium was already proposed by Eddington (1937) far before its actual spectral detection, which came almost four decades later when $\mathrm{H}_{2} \mathrm{O}$ ice was detected at $3 \mu \mathrm{m}$ by Gillett and Forrest (1973). Most ices in star-forming regions are observed in absorption in the mid-IR of an embedded object or along the line of sight of a background star. Ground based observations in the mid-IR spectral window are limited because of telluric absorptions, and mainly airborne and space observatories have been used to identify solid-phase species in space. Observations with the Infrared Space Observatory (ISO) have improved our understanding of interstellar ices. Because of its limited sensitivity, ISO observed mostly bright sources, such as high-mass young stellar objects (YSOs) and quiescent dense clouds toward luminous background stars (Gibb et al. 2000, 2004). More recently, the Spitzer space telescope characterized the molecular content of icy grain mantles in the 5$35 \mu \mathrm{m}$ wavelength range towards more than 40 low-mass protostars within the c2d (cores to disks) program (e.g., Boogert et al. 2008; Pontoppidan et al. 2008; Öberg et al. 2008; Bottinelli et al. 2010) and dozens more within other programs (Zasowski et al. 2009). This made it possible to unambiguously identify solid $\mathrm{H}_{2} \mathrm{O}, \mathrm{CO}_{2}, \mathrm{CH}_{4}, \mathrm{CH}_{3} \mathrm{OH}$, and $\mathrm{NH}_{3}$. Table 1 lists the main ice abundances with respect to $\mathrm{H}_{2} \mathrm{O}$ ice towards high- and low-mass protostars and quiescent dark clouds.

In quiescent dark clouds, interstellar grains provide a surface on which species can accrete, meet and react and to which they can donate excess energy. Grain-surface chemistry is governed by the accretion rate of gas-phase species onto the grains, the surface migration rate, which sets the reaction network, and the desorption rate. The timescale at which gas-phase species deplete-out onto grains is $\sim 10^{5}$ years in dense cores. This time is shorter than the lifetime of dense cores, which is between $10^{5}$ and $10^{6}$ years. 
Table 1 Interstellar ice feature inventory with respect to $\mathrm{H}_{2} \mathrm{O}$ ice towards dark clouds, low- and high-mass YSOs

\begin{tabular}{lllll}
\hline Species & $\lambda(\mu \mathrm{m})$ & Dark cloud (Elias 16) & L-m YSO (HH 46) & H-m YSO (W33A) \\
\hline $\mathrm{H}_{2} \mathrm{O}$ & 3.05 & 100 & 100 & 100 \\
$\mathrm{CO}$ & 4.67 & $26^{\mathrm{a}}$ & $20^{\mathrm{b}}$ & $8.1^{\mathrm{c}}$ \\
$\mathrm{CO}_{2}$ & 4.27 & $24^{\mathrm{d}}$ & $21.6^{\mathrm{e}}$ & $14.1^{\mathrm{e}}$ \\
$\mathrm{HCOOH}$ & 5.85 & $\leq 1.4^{\mathrm{f}}$ & $2.7^{\mathrm{f}}$ & $5.2^{\mathrm{f}}$ \\
$\mathrm{H}_{2} \mathrm{CO}$ & $3.47,3.54$ & $\ldots$ & $6.0^{\mathrm{f}}$ & $3.1^{\mathrm{c}}$ \\
$\mathrm{CH}_{3} \mathrm{OH}$ & 3.08 & $<2.3^{\mathrm{f}}$ & $6.1^{\mathrm{g}}$ & $14.7^{\mathrm{f}}$ \\
$\mathrm{NH}_{3}$ & 2.96 & $\leq 8^{\mathrm{d}}$ & $6.1^{\mathrm{g}}$ & $15^{\mathrm{c}}$ \\
$\mathrm{NH}_{4}^{+}$ & 6.85 & $5.2^{\mathrm{f}, \mathrm{i}}$ & $6.3^{\mathrm{f}, \mathrm{i}}$ & $8.1^{\mathrm{f}, \mathrm{i}}$ \\
$\mathrm{CH}_{4}$ & 3.32 & $<3^{\mathrm{d}}$ & $5.0^{\mathrm{h}}$ & $1.5^{\mathrm{c}}$ \\
$\mathrm{OCN}^{-}$ & 4.62 & $<2.3^{\mathrm{d}}$ & $\leq 0.6^{\mathrm{j}}$ & $1.9^{\mathrm{j}}$ \\
$\mathrm{OCS}$ & 4.92 & $<0.27^{\mathrm{c}}$ & $<0.04^{\mathrm{k}}$ & $0.2^{\mathrm{c}}$ \\
\hline
\end{tabular}

${ }^{a}$ Chiar et al. (1995)

b Boogert et al. (2004)

c Gibb et al. (2004)

d Knez et al. (2005)

e Pontoppidan et al. (2008)

f Boogert et al. (2008)

g Bottinelli et al. (2010)

h Öberg et al. (2008)

i The entire band is assumed to be due to $\mathrm{NH}_{4}^{+}$

$\mathrm{j}$ van Broekhuizen et al. (2005)

${ }^{\mathrm{k}}$ This value is taken from another low-mass YSO, Elias 29 (Gibb et al. 2004)

Hence, in dense regions, during the first stage of star formation virtually all species (except $\mathrm{H}_{2}$ ) are frozen-out onto interstellar grains. In this way, icy dust grains act both as a molecular reservoir and as a catalytic site. Subsequently, a complex grain-surface chemistry is triggered by photon/cosmic ray irradiation, thermal processing and particle bombardment (Herbst and van Dishoeck 2009).

Grain-surface chemistry dates back to Allen and Robinson (1977), and the first extended gas-grain model was proposed by Tielens and Hagen (1982). Their astrochemical model includes a complex grain surface reaction network to explain molecule formation in quiescent dark clouds. According to this model in a first phase, $\mathrm{H}_{2} \mathrm{O}$ ice (the dominant solidphase species) can be produced by the sequential hydrogenation of $\mathrm{O}$ atoms landing on the grain (a process initially proposed by van de Hulst; for a review see (van de Hulst 1996). Atomic oxygen can also react with other $\mathrm{O}$ atoms to form $\mathrm{O}_{2}$ and $\mathrm{O}_{3}$. Reaction of $\mathrm{O}_{3}$ with $\mathrm{H}$ reforms $\mathrm{O}_{2}$ and $\mathrm{OH} . \mathrm{O}_{2}$ can be hydrogenated to form $\mathrm{H}_{2} \mathrm{O}$ (through $\mathrm{H}_{2} \mathrm{O}_{2}$ ), while the $\mathrm{OH}$ radical can react with $\mathrm{H}$ or $\mathrm{H}_{2}$ to form again $\mathrm{H}_{2} \mathrm{O}$, or it can form $\mathrm{CO}_{2}$ and $\mathrm{H}_{2}$ with the $\mathrm{CO}$ accreted on the surface. Under these conditions, also other H-rich species such as $\mathrm{CH}_{4}$ and $\mathrm{NH}_{3}$ can be formed. Since hydrogen is the most abundant component of the interstellar gas, this first phase of grain-surface chemistry results in the formation of a polar ice (water-rich) mantle onto the dust grains. During the second phase, when the density increases in the molecular cloud, $\mathrm{CO}$ freezes-out onto the grains (its accretion rate is higher than that of $\mathrm{H}$ ) forming an apolar (water-poor) ice layer on top of the polar one. Under these conditions, 
the hydrogenation of $\mathrm{CO}$ ice leads to the formation of $\mathrm{H}_{2} \mathrm{CO}$ and $\mathrm{CH}_{3} \mathrm{OH}$ (Tielens and Hagen 1982; Charnley et al. 1997). $\mathrm{CO}_{2}$ can be formed through the reaction $\mathrm{CO}+\mathrm{OH}$ (Goumans et al. 2008). As suggested by Charnley et al. (2001), the ongoing reaction between $\mathrm{CO}$ or $\mathrm{CO}$ hydrogenation reaction products and elements heavier than $\mathrm{H}$ may lead to the formation of more complex molecules such as $\mathrm{CH}_{3} \mathrm{COH}$ and $\mathrm{C}_{2} \mathrm{H}_{5} \mathrm{OH}$ (see Fig. 1).

These and other astrochemical reaction networks were based on chemical intuition and analogs from gas-phase routes. It took several decades before experimental techniques allowed laboratory astrochemists to put all these reactions to the test. The laboratory studies presented in this review have a common bottom-up approach which aims to investigate at low temperatures selected and astrochemically relevant surface reactions, starting from the hydrogenation/ deuteration of pure and binary ices. The ultimate goal of this approach is to derive fundamental and molecule specific parameters, like reaction rates and diffusion barriers. In this experimental process, several of the previously proposed reactions were proven to be efficient, whereas others were not. Also several new reaction routes were revealed. The resulting experimentally measured reaction rates and diffusion barriers can then be included in astrochemical models which simulate ice evolution under

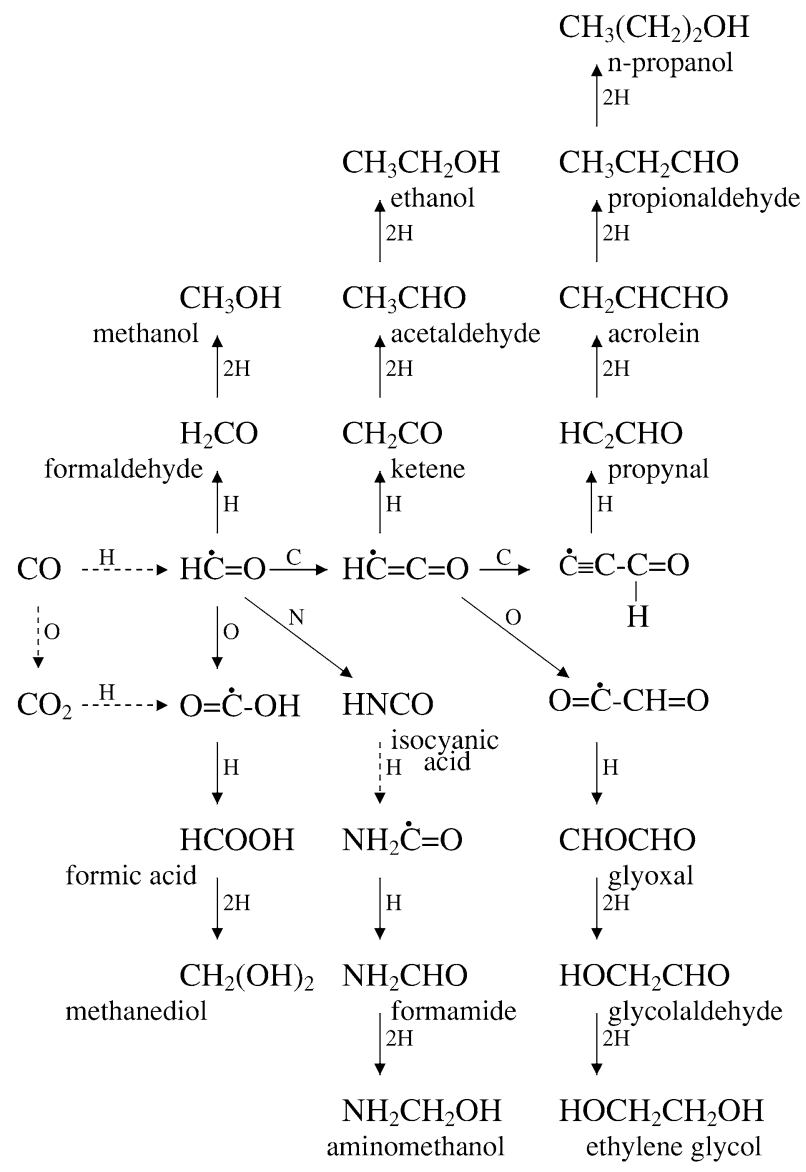

Fig. 1 Grain-surface atom addition reactions starting from CO as proposed by Charnley et al. (2001). Figure adapted from Charnley and Rodgers (2005) 
astronomical relevant timescales $\left(10^{5}\right.$ years $)$ and, therefore, push experimental results beyond typical laboratory timescales.

\section{Experimental}

All experiments presented here are performed using an ultra high vacuum (UHV) setup SURFace REaction SImulation DEvice (SURFRESIDE), which consists of a stainless steel vacuum main chamber and an atomic hydrogen line. A schematic view of the experimental apparatus is shown in Fig. 2. A gold coated copper substrate is mounted in the center of the UHV main chamber and its temperature is controlled between 12 and $300 \mathrm{~K}$. Deposition of selected gasses proceeds under an angle of $45^{\circ}$ with a controllable flow. Gas-phase molecules are monitored during the deposition mass spectrometrically by means of a quadrupole mass spectrometer (QMS), which is placed behind the substrate and opposite to the atomic source. A thermal cracking source (Tschersich and von Bonin 1998; Tschersich 2000; Tschersich et al. 2008) is used to generate $\mathrm{H}$ atoms through heating a capillary pipe, in which $\mathrm{H}_{2}$ flows, from 300 to $2,250 \mathrm{~K}$ by a surrounding tungsten filament. A quartz pipe is placed along the path of the dissociated beam to efficiently thermalize all $\mathrm{H}$ atoms to room temperature through surface collisions before they reach the ice sample. Atom fluxes produced by the hydrogen atom beam source (HABS) are measured quantitatively at the substrate position using the QMS, as described in the appendix of Ioppolo et al. (2010).

Ices are monitored by means of reflection-absorption infrared spectroscopy (RAIRS) using a Fourier transform infrared (FTIR) spectrometer, which covers the range between 4,000 and $700 \mathrm{~cm}^{-1}(2.5-14 \mu \mathrm{m})$. A spectral resolution between 1 and $4 \mathrm{~cm}^{-1}$ is used and several scans are co-added. In Fuchs et al. (2009), Ioppolo et al. (2008, 2010, 2011a) and Romanzin et al. (2011), the ice is first deposited and then hydrogenated/deuterated. In this case, RAIR difference spectra with respect to the initial deposited ice are acquired during H/D exposure. In Cuppen et al. (2010) the procedure is different and molecules are

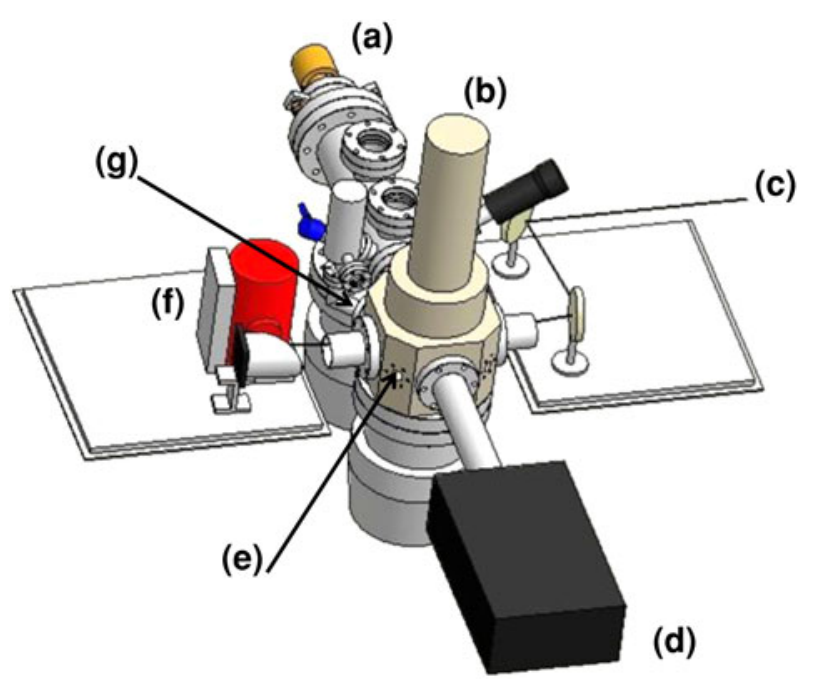

Fig. 2 Schematic top view of the solid-state experimental UHV set-up (SURFRESIDE): a H-atom source; b cold finger; c IR beam; $\mathbf{d}$ mass spectrometer; e main chamber; f IR detector; $\mathbf{g}$ deposition line 
co-deposited with $\mathrm{H}$ atoms. RAIR difference spectra are acquired with respect to the bare substrate during co-deposition. In all cases, newly formed solid species are monitored by RAIRS using unique IR spectral signatures. Spectra are recorded at different stages during hydrogenation, providing time resolved information about the destruction (i.e., use-up) of the precursor ice (the deposited ice layer) and the formation of new molecules that are identified through their spectral fingerprints. The intensity of a spectrum can be translated into a column density using a modified Lambert-Beer equation (Bennett et al. 2004). At the end of the $\mathrm{H}$-atom addition a temperature programmed desorption (TPD) experiment can be performed to constrain the spectroscopic results. Surface hydrogenation reactions of simple ices, like pure $\mathrm{CO}, \mathrm{O}_{2}, \mathrm{O}_{3}$, and $\mathrm{CO}: \mathrm{O}_{2}$ mixtures are investigated for a full range of different laboratory conditions including $\mathrm{H} / \mathrm{D}$-atom fluxes, ice temperatures, ice thicknesses, ice structures, and mixture ratios. This makes it possible to unravel the physics and chemistry of molecule formation and where applicable to examine in more detail the astronomical implications.

\section{Bottom-up versus top-down approach}

In the past the chemistry of inter- and circumstellar ice analogs has been studied using a top-down scenario: ice mixtures of astronomical constituents with more or less realistic mixing ratios were chemically triggered through UV/cosmic ray irradiation. The resulting residue was shown to consist of more complex organic compounds (e.g., Hagen et al. 1979; Allamandola et al. 1988; Gerakines et al. 1995; Hudson and Moore 2000; Strazzulla and Palumbo 2001; Mennella et al. 2004, 2006; Bennett and Kaiser 2007; Palumbo et al. 2008). The experimental results using this approach have been compared to interstellar ices generally in a more qualitative than quantitative way. More recently, a bottom-up approach has become experimentally possible, through the use of UHV setups in which individual reactions of simpler ices (i.e., not the cumulative outcome of a chemical network in an ice mixture) can be studied in situ and in real time under fully controlled laboratory conditions. This approach makes it possible to derive fundamental and molecule specific parameters, like reaction rates and diffusion barriers, which can then be included in astrochemical models to simulate the ice evolution under much longer timescales $\left(10^{5}\right.$ years) than accessible in the laboratory $(<1$ day)

The work presented in the next section follows a bottom-up approach and summarizes a representative sample of relevant experiments (e.g., Watanabe and Kouchi 2002; Watanabe et al. 2004, 2006; Fuchs et al. 2009; Miyauchi et al. 2008; Ioppolo et al. 2008, 2010, 2011a, b; Matar et al. 2008; Oba et al. 2009, 2010; Cuppen et al. 2010; Mokrane et al. 2009; Romanzin et al. 2011; Öberg et al. 2009). These experiments prove that species like $\mathrm{H}_{2} \mathrm{CO}, \mathrm{CH}_{3} \mathrm{OH}$ and $\mathrm{H}_{2} \mathrm{O}$ can be formed at low temperatures by simple hydrogenation (i.e., without the need for thermal, UV or cosmic ray processing) and provide the basic molecular data to simulate their formation on astronomical timescales (e.g., Cuppen et al. 2009), even though the ice as a whole is not representative for a realistic astronomical ice.

\section{Results}

Figure 3 shows the schematic representation of the reaction network that is presented here. The formation of formaldehyde and methanol ice through the $\mathrm{CO}+\mathrm{H}$ channel is shown on the left-side of the figure and discussed in Sect. 4.1, while the water formation channels 
Fig. 3 A schematic representation of the reaction network as discussed here. The $\mathrm{CO}+\mathrm{H}$ channel is shown on the left-side of the figure, while the $\mathrm{O} / \mathrm{O}_{2} / \mathrm{O}_{3}+\mathrm{H}$ channels are plotted on the right-side. The possible $\mathrm{CO}_{2}$ formation routes are shown in between the aforementioned channels. The figure is taken from Ioppolo et al. (2011a)

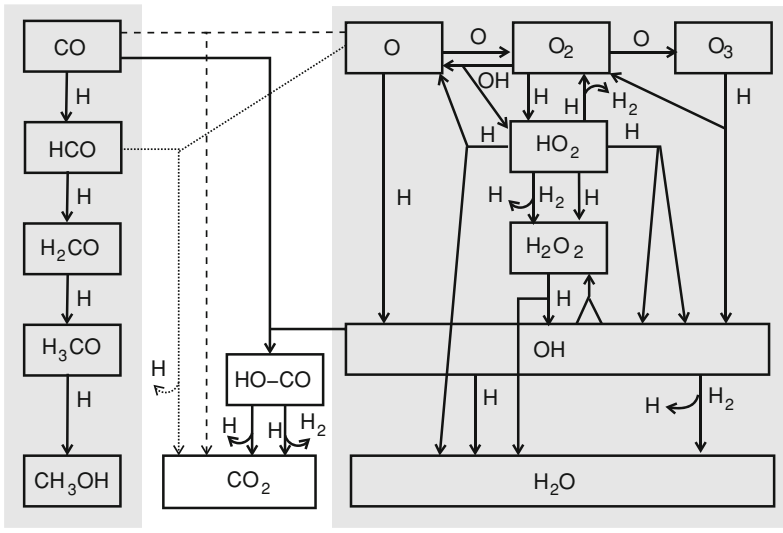

$\left(\mathrm{O} / \mathrm{O}_{2} / \mathrm{O}_{3}+\mathrm{H}\right)$ are plotted on the right-side and presented in Sect. 4.2. The possible $\mathrm{CO}_{2}$ formation routes are shown in between the aforementioned channels: the direct dissociation of the HO-CO intermediate (solid arrow) is discussed in Sect. 4.3; the hydrogenation of the HO-CO complex (solid arrow) is presented in Ioppolo et al. (2011b); the suggested $\mathrm{CO}+\mathrm{O}$ (dashed arrow) and $\mathrm{HCO}+\mathrm{O}$ (dotted arrow) routes have not been experimentally confirmed at low temperature (Tielens and Hagen 1982; Ruffle and Herbst 2001).

\subsection{Surface formation of methanol}

The surface formation of formaldehyde $\left(\mathrm{H}_{2} \mathrm{CO}\right)$ and methanol $\left(\mathrm{CH}_{3} \mathrm{OH}\right)$ by hydrogenation of $\mathrm{CO}$ ice has been topic of several studies (e.g., Hiraoka et al. 2002; Watanabe and Kouchi 2002; Fuchs et al. 2009). Methanol has been observed in interstellar ices and has been proposed as a good starting point for the formation of more complex species (Öberg et al. 2009). The hypothesis that interstellar methanol forms in the solid phase has been recently supported by experimental and modeling work, showing that the gas-phase route via ion-neutral reactions is less efficient than previously assumed (Geppert et al. 2005; Garrod et al. 2006). However, the first studies on solid CO hydrogenation by two different groups yielded conflicting results: in one study (Hiraoka et al. 2002) only the formation of $\mathrm{H}_{2} \mathrm{CO}$ was reported, whereas in the other study also $\mathrm{CH}_{3} \mathrm{OH}$ was observed (Watanabe and Kouchi 2002). The prevailing discrepancy between these studies was experimentally shown by Fuchs et al. (2009) to be a consequence of different experimental conditions, most noticeable the adopted H-atom flux (Hidaka et al. 2004). In Fuchs et al. (2009), reaction rates have been determined from RAIR data for different ice temperatures and ice thicknesses, as well as $\mathrm{H}$-atom fluxes (see Fig. 4). The formation of $\mathrm{H}_{2} \mathrm{CO}$ and $\mathrm{CH}_{3} \mathrm{OH}$ in the ice was confirmed spectrometrically. The experiments show that the hydrogenation process is thickness independent for layers thicker than four monolayers, which represents the maximum penetration depth of the $\mathrm{H}$ atoms into the $\mathrm{CO}$ ice. For temperatures higher than $15 \mathrm{~K}$, a clear drop in the production rate of methanol is observed. This is probably due to the reduced residence-time of $\mathrm{H}$ atoms into the ice at those temperatures, which decreases the probability that hydrogenation reactions occur. Since the rate of formation of molecules depends on a sequence of events: deposition of $\mathrm{H}$ atoms, diffusion and then reaction, reaction barriers can only be determined using a detailed model to fit the experimental data (e.g., Fuchs et al. 2009; Cuppen et al. 2009). These results can then be 


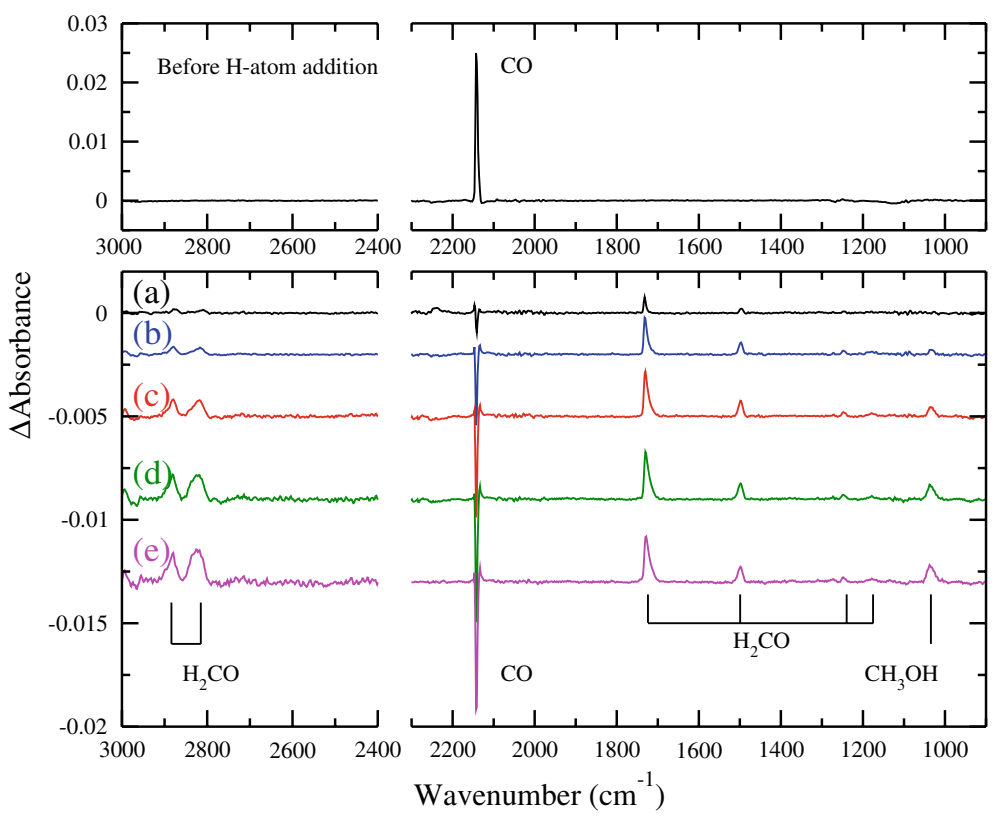

Fig. 4 The RAIR difference spectra of a CO + H experiment (Fuchs et al. 2009). The top panel shows the CO ice spectrum at $T=15 \mathrm{~K}$. The bottom panel shows the RAIR difference spectra with respect to the initial deposited ice acquired during $\mathrm{H}$-atom exposure at $T=15 \mathrm{~K}$ for increasing fluences $(a-e)$. The use-up of $\mathrm{CO}$ and formation of $\mathrm{H}_{2} \mathrm{CO}$ and $\mathrm{CH}_{3} \mathrm{OH}$ are clearly seen

used in an astrochemical model to investigate the formation of formaldehyde and methanol under interstellar conditions (see Sect. 5).

\subsection{Surface formation of water}

Tielens and Hagen (1982) proposed that interstellar water forms on grain surfaces through three reaction channels: hydrogenation of atomic, molecular oxygen and ozone. Using a Monte Carlo approach, Cuppen and Herbst (2007) and Cazaux et al. (2010) showed that the contribution of the different formation channels strongly depends on the local environment in interstellar clouds. They concluded that the atomic oxygen channel is the main route in translucent and diffuse clouds, while the molecular oxygen channel, together with the ozone route, is more efficient in dense cold molecular clouds.

Water ice formation through surface reactions has been studied in detail (see Fig. 5). All three hydrogenation channels have been investigated qualitatively by Dulieu et al. (2010) in the submonolayer regime using TPD as their main analysis technique, confirming the formation of water ice. The hydrogenation of solid $\mathrm{O}_{2}$ is the most extensively studied channel (e.g., Miyauchi et al. 2008; Ioppolo et al. 2008; Matar et al. 2008). Ioppolo et al. (2008) investigated this reaction channel for a large range of astronomically relevant temperatures $(12-28 \mathrm{~K})$. The main and surprising finding is that the initial formation rate of $\mathrm{H}_{2} \mathrm{O}_{2}$ and $\mathrm{H}_{2} \mathrm{O}$ is much less temperature dependent than the analogous reactions for $\mathrm{CO}$ hydrogenation, as discussed above. Furthermore, $\mathrm{O}_{2}$ hydrogenation results in a much larger yield than the few monolayers found for $\mathrm{CO}$ hydrogenation. This yield is strongly temperature dependent. Both effects are shown to be a direct consequence of the ability of $\mathrm{H}$ 

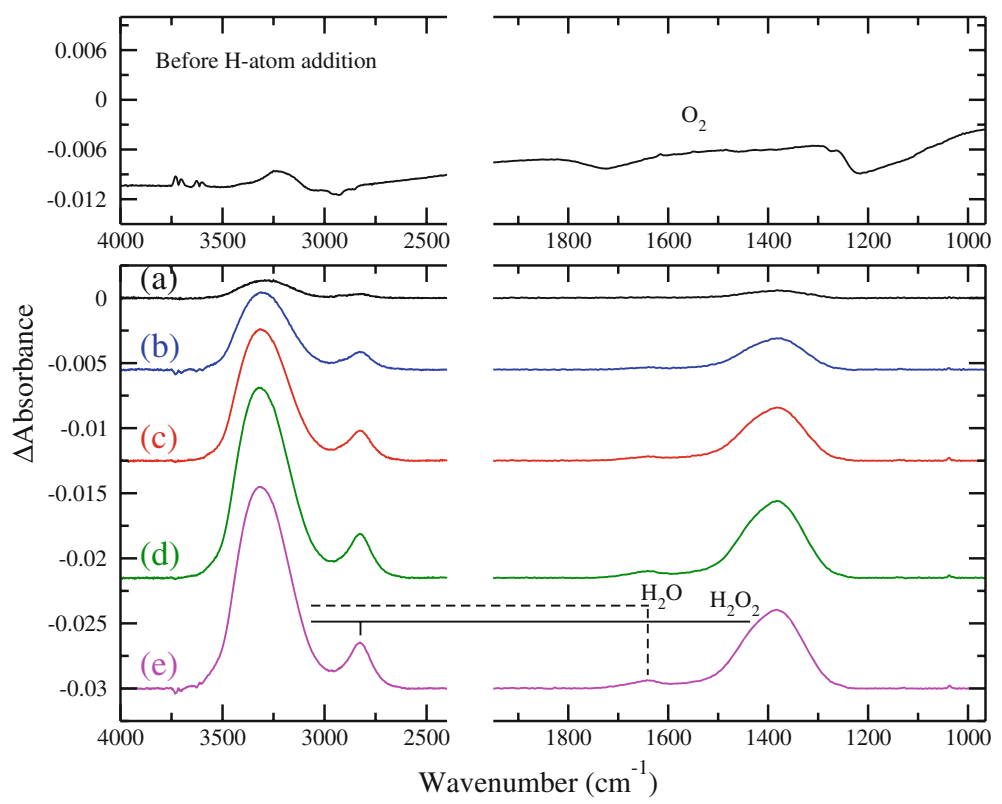

Fig. 5 The RAIR difference spectra of an $\mathrm{O}_{2}+\mathrm{H}$ experiment (Ioppolo et al. 2010). The top panel shows the $\mathrm{O}_{2}$ ice spectrum at $T=15 \mathrm{~K}$. The bottom panel shows the RAIR difference spectra with respect to the initial deposited ice acquired during $\mathrm{H}$-atom exposure at $T=25 \mathrm{~K}$ for increasing fluences $(a-e)$. The formation of $\mathrm{H}_{2} \mathrm{O}_{2}$ and $\mathrm{H}_{2} \mathrm{O}$ is clearly shown

atoms to diffuse easily through an $\mathrm{O}_{2}$ ice (Ioppolo et al. 2010). The experiments show that the penetration depth of $\mathrm{H}$ atoms into the ice depends strongly on the ice composition. In Ioppolo et al. (2010), $\mathrm{O}_{2}$ hydrogenation is investigated extensively from a physical approach, i.e., studying different ice thickness, ice temperature, ice structure and $\mathrm{H}_{2}$ concentration in the atomic beam, whereas in a follow-up paper (Cuppen et al. 2010), the focus is on the reaction scheme with the intent to assess reaction routes and branching ratios. The latter work shows that the initially proposed reaction network (Tielens and Hagen 1982) of only three mainly isolated channels is too simple and that several of the channels are actually linked through additional reactions.

The third water formation channel (the hydrogenation of solid $\mathrm{O}_{3}$ ) was tested by Mokrane et al. (2009) and more recently by Romanzin et al. (2011). Since this channel is connected to the $\mathrm{O}_{2}$ channel after the first reaction step, special care was taken in Romanzin et al. (2011) to deposit a pure $\mathrm{O}_{3}$ ice by keeping the substrate temperature between the $\mathrm{O}_{2}$ and $\mathrm{O}_{3}$ desorption temperature during deposition. If such a temperature is also kept during $\mathrm{H}$-atom addition, the $\mathrm{O}_{2}$ molecules formed upon $\mathrm{O}_{3}$ hydrogenation will desorb from the surface of the ice. In this way the reaction of $\mathrm{OH}$ to form water via $\mathrm{H}$ or $\mathrm{H}_{2}$ addition can be probed. The hydrogenation of $\mathrm{O}_{3}$ is found to behave more similar to $\mathrm{CO}$ hydrogenation in the sense that only the top few monolayers of $\mathrm{O}_{3}$ are hydrogenated. Moreover, the reaction $\mathrm{OH}+\mathrm{H}_{2}$ may be more efficient than the reaction $\mathrm{OH}+\mathrm{H}$ : reaction $\mathrm{OH}+\mathrm{H}_{2}$ likely proceeds through tunneling, while reaction $\mathrm{OH}+\mathrm{H}$ needs to dissipate $5.3 \mathrm{eV}$ of excess energy with just one final product, which could be difficult.

These experimental results complete the reaction scheme on water formation initially proposed (Tielens and Hagen 1982). The general conclusion that the three channels $\left(\mathrm{O} / \mathrm{O}_{2} /\right.$ 
$\mathrm{O}_{3}+\mathrm{H}$ ) are strongly linked, is of importance for astrochemical models focusing on water formation under interstellar conditions (Wakelam et al. 2010).

\subsection{Surface formation of $\mathrm{CO}_{2}$}

Ioppolo et al. (2011a) proved the surface formation of $\mathrm{CO}_{2}$ at low temperatures through the reaction $\mathrm{CO}+\mathrm{OH}$ and subsequent dissociation of the resulting $\mathrm{HO}-\mathrm{CO}$ complex by hydrogenation of a $\mathrm{CO}: \mathrm{O}_{2}$ ice mixture (see Fig. 6). Such a binary ice is not fully representative for an interstellar ice, but the hydrogenation of $\mathrm{O}_{2}$ ice produces $\mathrm{OH}$ radicals, which allows the investigation of the interstellar relevant $\mathrm{CO}+\mathrm{OH}$ solid state reaction. As discussed above similar astrophysical ice studies have focused on the investigation of isolated surface reaction schemes, starting from the hydrogenation of pure ices, like solid $\mathrm{CO}$ and $\mathrm{O}_{2}$. For such ices no $\mathrm{CO}_{2}$ formation was observed upon $\mathrm{H}$-atom exposure. The hydrogenation of binary ice mixtures allows to investigate the influence of the presence of other species in the ice on the pure ice reaction schemes. Mixtures of $\mathrm{CO}: \mathrm{O}_{2}$ are deposited on a substrate under ultra high vacuum conditions at low temperatures ( 15 and $20 \mathrm{~K})$ and subsequently hydrogenated. The RAIRS shows that solid $\mathrm{CO}_{2}$ is formed in all studied $\mathrm{CO}: \mathrm{O}_{2}$ mixtures. Within the experimental uncertainties no dependency on ice temperature or composition was observed. The competition between $\mathrm{CO}$ hydrogenation and $\mathrm{O}_{2}$ hydrogenation in the mixed ice reveals that the penetration depth of $\mathrm{H}$ atoms into the ice depends strongly on the ice composition, and that the $\mathrm{CO}$ and $\mathrm{O}_{2}$ channels influence each others final product yields. The formation rate for all the final products is found to be less

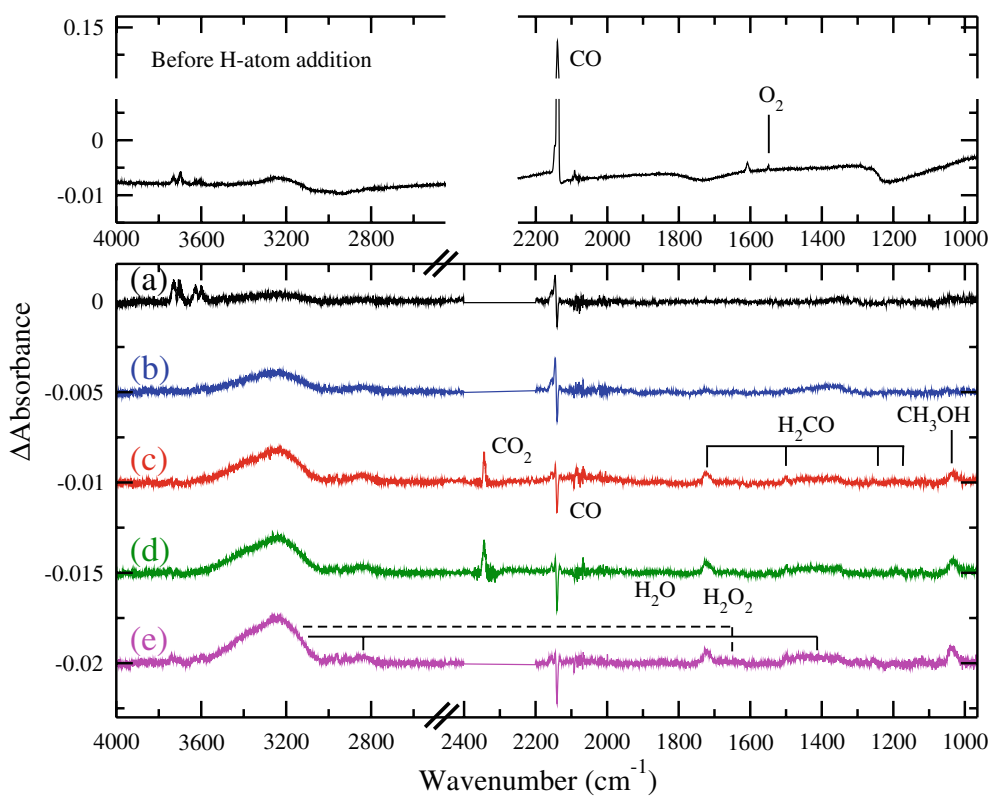

Fig. 6 The RAIR difference spectra of a CO:O $\mathrm{O}_{2}+\mathrm{H}$ experiment (Ioppolo et al. 2011a). The top panel shows the $\mathrm{CO}: \mathrm{O}_{2}=1: 1$ mixed ice spectrum at $T=15 \mathrm{~K}$. The bottom panel shows the RAIR difference spectra with respect to the initial deposited ice acquired during $\mathrm{H}$-atom exposure at $T=15 \mathrm{~K}$ for increasing fluences $(a-e)$. The formation of solid $\mathrm{CO}_{2}$ is clearly shown in spectra $c$ and $d$ at $\sim 2340 \mathrm{~cm}^{-1}$. The solid $\mathrm{CO}_{2}$ peak is not plotted in spectra $a, b$, and $e$ because gas-phase $\mathrm{CO}_{2}$, which is present outside the UHV chamber and in the pathway of the IR beam, covers the solid $2340 \mathrm{~cm}^{-1}$ band 
sensitive on the mixture composition than the final yield. Therefore, the formation rates found for $\mathrm{H}_{2} \mathrm{CO}, \mathrm{CH}_{3} \mathrm{OH}, \mathrm{H}_{2} \mathrm{O}_{2}$ and $\mathrm{H}_{2} \mathrm{O}$ in the isolated studies of the $\mathrm{CO}+\mathrm{H}$ (Fuchs et al. 2009) and $\mathrm{O}_{2}+\mathrm{H}$ (Ioppolo et al. 2008) channels are valid for use in astrochemical models. In addition, surface $\mathrm{CO}_{2}$ formation without energetic input is found to be an important formation mechanism, which may explain the formation of $\mathrm{CO}_{2}$ together with $\mathrm{H}_{2} \mathrm{O}$ ice during the dense cold core phase prior to star formation.

\section{Extending the laboratory data to ISM conditions}

The conditions in the experiments discussed in the previous sections do not fully reproduce the conditions in space. Although temperature, pressure, thickness and substrate, can be approached quite accurately, the experimental particle flux can never be close to the flux in the ISM due to timescale issues. Typically, fluences are reached within a few hours in the laboratory that are similar to interstellar fluences after a million years.

For multiple step processes such as surface reactions, which are the result of a sequence of diffusion and reaction events, the product yield does not necessarily scale with the fluence. The separate steps need to be disentangled and characterized by rates. The ultimate goal of the bottom-up experiments applied here is, therefore, to obtain physico-chemical parameters such as reaction rates that can be included in astrochemical models to simulate the chemical evolution of different astrophysical objects under a range of different physical conditions.

One way of doing this is by first simulating the experimental conditions using these input parameters as fitting parameters. The same simulation routine can then be applied to simulate the chemical evolution under interstellar conditions. Using continuous-time, random-walk (CTRW-) Monte Carlo simulations this has be done for methanol formation. First, the experimental conditions have been simulated using the reaction rates of $\mathrm{H}+\mathrm{CO}$ and $\mathrm{H}+\mathrm{H}_{2} \mathrm{CO}$ as fitting parameters (Fuchs et al. 2009). The Monte Carlo simulation were found in good agreement with the experimental data especially at low temperatures, where the laboratory results are less affected by experimental limits like the presence of $\mathrm{H}_{2}$ molecules in the $\mathrm{H}$-atom beam or the lower sticking probability of the thermal $\mathrm{H}$ atoms $(300 \mathrm{~K})$ at higher substrate temperatures. The resulting values were then applied to simulate formaldehyde and methanol formation in cold dense cores (Cuppen et al. 2009).

The advantage of using CTRW-Monte Carlo simulations is that species can be followed on the surface. Layering is, therefore, automatically taken into account. This is important as only the top layers are affected and the atoms do not penetrate deeply into the solid. The simulations of the experiments showed that the production rate of formaldehyde decreases and that the penetration depth into the ice increases with temperature.

Simulations for different interstellar parameters, including density and temperature, have been performed in Cuppen et al. (2009). Formaldehyde and methanol were found to form efficiently in cold dense cores or the cold outer envelopes of young stellar objects. Again layering plays an important role, since the grain mantle is found to have a layered structure with $\mathrm{CH}_{3} \mathrm{OH}$ on top at the end of the freeze-up time. The species $\mathrm{CO}$ and $\mathrm{H}_{2} \mathrm{CO}$ are found to exist predominantly in the lower layers of ice mantles where they are not available for hydrogenation at late times. This finding is in contrast with previous gas-grain models, which do not take into account the layering of the ice. Observational solid $\mathrm{H}_{2} \mathrm{CO} /$ $\mathrm{CH}_{3} \mathrm{OH}$ and $\mathrm{CO} / \mathrm{CH}_{3} \mathrm{OH}$ abundance ratios in the outer envelopes of an assortment of young stellar objects agree reasonably well with our model results, which also suggests that 
the large range in $\mathrm{CH}_{3} \mathrm{OH} / \mathrm{H}_{2} \mathrm{O}$ observed abundance ratios is due to variation in the evolutionary stages of the selected YSOs.

\section{Conclusions}

In this overview the surface formation of $\mathrm{H}_{2} \mathrm{CO}, \mathrm{CH}_{3} \mathrm{OH}, \mathrm{H}_{2} \mathrm{O}$, and $\mathrm{CO}_{2}$ at low temperatures is experimentally shown using a bottom-up approach, in which individual surface reactions are experimentally investigated starting from the hydrogenation of simple and binary ices (i.e., pure $\mathrm{CO}, \mathrm{O}_{2}, \mathrm{O}_{3}$ ices and mixed $\mathrm{CO}: \mathrm{O}_{2}$ ices). These studies prove that molecules like methanol, water and carbon dioxide can be formed in the solid state in the ISM without the need for energetic processing such as thermal, UV/cosmic ray processing (i.e., through $\mathrm{H}$-atom addition). Here, several of the formation routes proposed in the past by astrochemical models based on gas-phase data were proven to be efficient, whereas others were not. Also several new reaction routes were revealed. The experimental outcome can be used in astrochemical models with the intent to simulate the formation of new species in the solid phase on astronomical timescales (e.g., Cuppen et al. 2009), extending the laboratory results beyond experimental constraints.

Acknowledgments The research leading to these results has received funding from NOVA, the Netherlands Research School for Astronomy and the European Community's Seventh Framework Programme (FP7/2007-2013) under grant agreement no. 238258.

Open Access This article is distributed under the terms of the Creative Commons Attribution Noncommercial License which permits any noncommercial use, distribution, and reproduction in any medium, provided the original author(s) and source are credited.

\section{References}

Allamandola LJ, Sandford SA, Valero GJ (1988) Photochemical and thermal evolution of interstellar/ precometary ice analogs. Icarus 76:225

Allen M, Robinson GW (1977) The molecular composition of dense interstellar clouds. Astrophys J 212:396

Bennett CJ, Kaiser RI (2007) On the formation of glycolaldehyde $\left(\mathrm{HCOCH}_{2} \mathrm{OH}\right)$ and methyl formate $\left(\mathrm{HCOOCH}_{3}\right)$ in interstellar ice analogs. Astrophys J 661:899

Bennett CJ, Jamieson C, Mebel AM, Kaiser RI (2004) Untangling the formation of the cyclic carbon trioxide isomer in low temperature carbon dioxide ices. Phys Chem Chem Phys 6:735

Boogert ACA, Pontoppidan KM, Lahuis F et al (2004) Spitzer space telescope spectroscopy of ices toward low-mass embedded protostars. Astrophys J Suppl 154:359

Boogert ACA, Pontoppidan KM, Knez C et al (2008) The c2d Spitzer spectroscopic survey of ices around low-mass young stellar objects. $\mathrm{I}$. $\mathrm{H}_{2} \mathrm{O}$ and the 5-8 m bands. Astrophys J 678:985

Bottinelli S, Boogert AC, Bouwman J et al (2010) The c2d Spitzer spectroscopic survey of ices around lowmass young stellar objects. IV. $\mathrm{NH}_{3}$ and $\mathrm{CH}_{3} \mathrm{OH}$. Astrophys $\mathrm{J}$ 718:1100

Cami J, Bernard-Salas J, Peeters E, Malek SE (2010) Detection of $\mathrm{C}_{60}$ and $\mathrm{C}_{70}$ in a young planetary nebula. Science 329:1180

Cazaux S, Cobut V, Marseille M, Spaans M, Caselli P (2010) Water formation on bare grains: when the chemistry on dust impacts interstellar gas. Astron Astrophys 522:A74

Charnley SB, Rodgers SD (2005) Pathways to molecular complexity. In: Proceedings of IAU Symposium on Astrochemistry: recent successes and current challenges, vol 231, The Netherlands

Charnley SB, Tielens AGGM, Rodgers SD (1997) Deuterated methanol in the Orion compact ridge. Astrophys J 482:L203

Charnley SB, Rodgers SD, Ehrenfreund P (2001) Gas-grain chemical models of star-forming molecular clouds as constrained by ISO and SWAS observations. Astron Astrophys 378:1024

Chiar JE, Adamson AJ, Kerr TH, Whittet DCB (1995) High-resolution studies of solid CO in the taurus dark cloud: characterizing the ices in quiescent clouds. Astrophys J 455:234 
Cuppen HM, Herbst E (2007) Simulation of the formation and morphology of ice mantles on interstellar grains. Astrophys J 668:294

Cuppen HM, van Dishoeck EF, Herbst E, Tielens AGGM (2009) Microscopic simulation of methanol and formaldehyde ice formation in cold dense cores. Astron Astrophys 508:275

Cuppen HM, Ioppolo S, Romanzin C, Linnartz H (2010) Water formation at low temperatures by surface $\mathrm{O}_{2}$ hydrogenation II: the reaction network. Phys Chem Chem Phys 12:12077

Dulieu F, Amiaud L, Congiu E, Fillion J, Matar E, Momeni A, Pirronello V, Lemaire JL (2010) Experimental evidence for water formation on interstellar dust grains by hydrogen and oxygen atoms. Astron Astrophys 512:A30

Eddington AS (1937) Interstellar matter. The Observatory 60:99

Fuchs GW, Cuppen HM, Ioppolo S, Bisschop SE, Andersson S, van Dishoeck EF, Linnartz H (2009) Hydrogenation reactions in interstellar $\mathrm{CO}$ ice analogues. A combined experimental/theoretical approach. Astron Astrophys 505:629

Garrod R, Park IH, Caselli P, Herbst E (2006) Are gas-phase models of interstellar chemistry tenable? The case of methanol. Faraday Discuss 133:5

Geppert WD, Thomas RD, Ehlerding A et al (2005) Dissociative recombination branching ratios and their influence on interstellar clouds. J Phys Conf Ser 4:26

Gerakines PA, Schutte WA, Greenberg JM, van Dishoeck EF (1995) The infrared band strengths of $\mathrm{H}_{2} \mathrm{O}$, $\mathrm{CO}$ and $\mathrm{CO}_{2}$ in laboratory simulations of astrophysical ice mixtures. Astron Astrophys 296:810

Gibb EL, Whittet DCB, Schutte WA et al (2000) An inventory of interstellar ices toward the embedded protostar W33A. Astrophys J 536:347

Gibb EL, Whittet DCB, Boogert ACA, Tielens AGGM (2004) Interstellar ice: the infrared space observatory legacy. Astrophys J Suppl 151:35

Gillett FC, Forrest WJ (1973) Spectra of the Becklin-Neugebauer point source and the Kleinmann-Low nebula from 2.8 to 13.5 microns. Astrophys J 179:483

Goumans TPM, Uppal MA, Brown WA (2008) Formation of $\mathrm{CO}_{2}$ on a carbonaceous surface: a quantum chemical study. Mon Not R Astron Soc 384:1158

Hagen W, Allamandola LJ, Greenberg JM (1979) Interstellar molecule formation in grain mantles-the laboratory analog experiments, results and implications. Astrophys Space Sci 65:215

Herbst E, van Dishoeck EF (2009) Complex organic interstellar molecules. Annu Rev Astron Astrophys 47:427

Hidaka H, Watanabe N, Shiraki T, Nagaoka A, Kouchi A (2004) Conversion of $\mathrm{H}_{2} \mathrm{CO}$ to $\mathrm{CH}_{3} \mathrm{OH}$ by reactions of cold atomic hydrogen on ice surfaces below $20 \mathrm{~K}$. Astrophys J 614:1124

Hiraoka K, Sato T, Sato S, Sogoshi N, Yokoyama T, Takashima H, Kitagawa S (2002) Formation of formaldehyde by the tunneling reaction of $\mathrm{H}$ with solid $\mathrm{CO}$ at $10 \mathrm{~K}$ revisited. Astrophys $\mathrm{J}$ 577:265

Hudson RL, Moore MH (2000) New experiments and interpretations concerning the "XCN" band in interstellar ice analogues. Astron Astrophys 357:787

Ioppolo S, Cuppen HM, Romanzin C, van Dishoeck EF, Linnartz H (2008) Laboratory evidence for efficient water formation in interstellar ices. Astrophys J 686:1474

Ioppolo S, Cuppen HM, Romanzin C, van Dishoeck EF, Linnartz H (2010) Water formation at low temperatures by surface $\mathrm{O}_{2}$ hydrogenation $\mathrm{I}$ : characterization of ice penetration. Phys Chem Chem Phys 12:12065

Ioppolo S, van Boheemen Y, Cuppen HM, van Dishoeck EF, Linnartz H (2011a) Surface formation of $\mathrm{CO}_{2}$ ice at low temperatures. Mon Not R Astron Soc 413:2281

Ioppolo S, Cuppen HM, van Dishoeck EF, Linnartz H (2011b) Surface formation of HCOOH at low temperature. Mon Not R Astron Soc 410:1089

Knez C, Boogert ACA, Pontoppidan KM et al (2005) Spitzer mid-infrared spectroscopy of ices toward extincted background stars. Astrophys J Lett 635:L145

Matar E, Congiu E, Dulieu F, Momeni A, Lemaire JL (2008) Mobility of D atoms on porous amorphous water ice surfaces under interstellar conditions. Astron Astrophys 492:L17

Mennella V, Palumbo ME, Baratta GA (2004) Formation of $\mathrm{CO}$ and $\mathrm{CO}_{2}$ molecules by ion irradiation of water ice-covered hydrogenated carbon grains. Astrophys J 615:1073

Mennella V, Baratta GA, Palumbo ME, Bergin EA (2006) Synthesis of CO and $\mathrm{CO}_{2}$ molecules by UV irradiation of water ice-covered hydrogenated carbon grains. Astrophys J 643:923

Miyauchi N, Hidaka H, Chigai T, Nagaoka A, Watanabe N, Kouchi A (2008) Formation of hydrogen peroxide and water from the reaction of cold hydrogen atoms with solid oxygen at $10 \mathrm{~K}$. Chem Phys Lett 456:27

Mokrane H, Chaabouni H, Accolla M, Congiu E, Dulieu F, Chehrouri M, Lemaire JL (2009) Experimental evidence for water formation via ozone hydrogenation on dust grains at $10 \mathrm{~K}$. Astrophys $\mathrm{J}$ Lett 705:L195 
Oba Y, Miyauchi N, Hidaka H, Chigai T, Watanabe N, Kouchi A (2009) Formation of compact amorphous $\mathrm{H}_{2} \mathrm{O}$ ice by codeposition of hydrogen atoms with oxygen molecules on grain surfaces. Astrophys. J $701: 464$

Oba Y, Watanabe N, Kouchi A, Hama T, Pirronello V (2010) Experimental study of $\mathrm{CO}_{2}$ formation by surface reactions of non-energetic $\mathrm{OH}$ radicals with $\mathrm{CO}$ molecules. Astrophys J Lett 712:L174

Öberg KI, Boogert ACA, Pontoppidan KM, Blake GA, Evans NJ, Lahuis F, van Dishoeck EF (2008) The c2d Spitzer spectroscopic survey of ices around low-mass young stellar objects. III. $\mathrm{CH}_{4}$. Astrophys $\mathbf{J}$ 4(678): 1032

Öberg KI, Garrod RT, van Dishoeck EF, Linnartz H (2009) Formation rates of complex organics in UV irradiated $\mathrm{CH}_{3} \mathrm{OH}$-rich ices. I. Experiments. Astron Astrophys 504:891

Palumbo ME, Baratta GA, Fulvio D, Garozzo M, Gomis O, Leto G, Spinella F, Strazzulla G (2008) Ion irradiation of astrophysical ices. J Phys Conf Ser 101:012002

Pontoppidan KM, Boogert ACA, Fraser HJ et al (2008) The c2d Spitzer spectroscopic survey of ices around low-mass young stellar objects. II. $\mathrm{CO}_{2}$. Astrophys J 678:1005

Romanzin C, Ioppolo S, Cuppen HM, van Dishoeck EF, Linnartz H (2011) Water formation by surface $\mathrm{O}_{3}$ hydrogenation. J Chem Phys 134:084504

Ruffle DP, Herbst E (2001) New models of interstellar gas-grain chemistry-II. Surface photochemistry in quiescent cores. Mon Not R Astron Soc 322:770

Sellgren K, Werner MW, Ingalls JG, Smith JDT, Carleton TM, Joblin C (2010) $\mathrm{C}_{60}$ in reflection nebulae. Astrophys. J. Lett 722:L54

Strazzulla G, Palumbo ME (2001) Organics produced by ion irradiation of ices: some recent results. Adv Space Res 27:237

Tielens AGG (2005) The Physics and Chemistry of the Interstellar Medium. Cambridge University Press, New York

Tielens AGGM, Hagen W (1982) Model calculations of the molecular composition of interstellar grain mantles. Astron Astrophys 114:245

Tschersich KG (2000) Intensity of a source of atomic hydrogen based on a hot capillary. J Appl Phys 87:2565

Tschersich KG, von Bonin V (1998) Formation of an atomic hydrogen beam by a hot capillary. J Appl Phys 84:4065

Tschersich KG, Fleischhauer JP, Schuler H (2008) Design and characterization of a thermal hydrogen atom source. J Appl Phys 104:034908

Wakelam V, Smith IWM, Herbst E et al (2010) Reaction networks for interstellar chemical modelling: improvements and challenges. Space Sci Rev 156:13

Watanabe N, Kouchi A (2002) Efficient formation of formaldehyde and methanol by the addition of hydrogen atoms to $\mathrm{CO}$ in $\mathrm{H}_{2} \mathrm{O}-\mathrm{CO}$ ice at $10 \mathrm{~K}$. Astrophys J Lett 571:L173

Watanabe N, Nagaoka A, Shiraki T, Kouchi A (2004) Hydrogenation of CO on pure solid CO and CO- $\mathrm{H}_{2} \mathrm{O}$ mixed ice. Astrophys J 616:638

Watanabe N, Hidaka H, Kouchi A (2006) Astrochemistry-from laboratory studies to astronomical observations. AIP Conf Ser 855:122

van Broekhuizen FA, Pontoppidan KM, Fraser HJ, van Dishoeck EF (2005) A 3-5 m VLT spectroscopic survey of embedded young low mass stars II. Solid $\mathrm{OCN}^{-}$. Astron Astrophys 441:249

van de Hulst HC (1996) Molecules in astrophysics half a century ago. In: Proceedings of IAU symposium on molecules in astrophysics: probes \& processes, vol 178, The Netherlands

Zasowski G, Kemper F, Watson DM, Furlan E, Bohac CJ, Hull C, Green JD (2009) Spitzer infrared spectrograph observations of class I/II objects in taurus: composition and thermal history of the circumstellar ices. Astrophys J 694:459 